Pacific Journal of Mathematics

AN APPLICATION OF WERMER'S SUBHARMONICITY 


\section{AN APPLICATION OF WERMER'S SUBHARMONICITY THEOREM}

\section{GLICKSBERG}

Wermer originally introduced his subharmonicity theorem to find analytic structure in certain polynomial hulls in $C^{2}$ and later, with Aupetit, applied it to improve a well known result of Bishop providing analytic structure in the spectra of certain uniform algebras. We make a simpler application to yield analytic structure in the presence of an element of constant modulus on certain fibers provided by another element, and to extend Wermer's maximality theorem.

1. Let $A$ be a uniform algebra [4] on its spectrum $M=M_{A}$, with $X$ a boundary for $A$. Our basic observation is contained in the simple

LemMA 1.1. Suppose $g \in A, f \in A^{-1}$ and

$$
\text { for each } \zeta \in g(X),\left|f\left(g^{-1}(\zeta)\right)\right| \text { is a singleton. }
$$

Then the same is true for any $\zeta$ in $g(M)$ and

$$
u: \zeta \longrightarrow \log \left|f\left(g^{-1}(\zeta)\right)\right|
$$

is continuous on $g(M)$ and harmonic on $g(M) \backslash g(X)$.

This consequence of Wermer's theorem yields our application, which in its simplest form is

THEOREM 1.2. Suppose $g \in A$ and $f \in A^{-1}$ satisfy (1) and separate M. If $U$ is a simply connected component of $g(M) \backslash g(X)$ then $g^{-1}(U)$ can be viewed as a product of $U$ and a closed subset $K$ of the circle $T^{1}=\{|z|=1\}$ with $U \times\{z\}$ an "analytic disc"; more precisely, for any subdomain $V \subset U, g^{-1}\left(V^{-}\right)$is topologically equivalent to $V^{-} \times K$, and $\left(A \mid g^{-1}\left(V^{-}\right)\right)^{-}$, transported to $V^{-} \times K$, consists of functions with sections analytic on $V$.

When $U$ is not simply connected $g^{-1}(U)$ can be viewed as a product of a (not necessarily closed) subset $K$ of the circle and a Riemann surface as we shall see, but our correspondence need not be topological. When $f$ and $g$ do not separate $M$ we can apply our argument to provide such analytic structure in the part of the joint spectrum $\sigma_{A}(g, f)$ of $g, f$ over $U, \pi_{1}^{-1}(U) \cap \sigma_{A}(g, f)$, or of the joint spectrum $\sigma_{B}(g, f)$ relative to any subalgebra $B$ containing that 
generated by $g, f$ and $f^{-1}$, provided we have some way of insuring (1) applies when $g^{-1}(\zeta)$ is replaced by $\hat{g}^{-1}(\zeta)$, the fiber in $M_{B}$ (which can be different, of course). In particular this is the case if $g(X)$ is the minimal boundary for $R(g(M))$, so $\hat{g}^{-1}(\zeta) \subset M_{B}$ is a peak set for $B$, and consequently $B \mid \hat{g}^{-1}(\zeta)$ is a closed subalgebra of $C\left(\hat{g}^{-1}(\zeta)\right)$ with $\hat{g}^{-1}(\zeta) \cap X=g^{-1}(\zeta) \cap X$ a boundary ${ }^{1)}$, so

$$
\sup \left|\hat{f}\left(\hat{g}^{-1}(\zeta)\right)\right| \leqq \sup \left|f\left(g^{-1}(\zeta)\right)\right|
$$

while

$$
\inf \left|\widehat{f}\left(\hat{g}^{-1}(\zeta)\right)\right| \geqq \inf \left|f\left(g^{-1}(\zeta)\right)\right|
$$

(since $f$ is invertible), and thus $\left|\hat{f}\left(\hat{g}^{-1}(\zeta)\right)\right|$ coincides with the singleton $\left|f\left(g^{-1}(\zeta)\right)\right|$.

Corollary 1.3. Suppose $g \in A$ and $f \in A^{-1}$ satisfy (1), $g(X)$ is the minimal boundary for $R(g(M))$, and $B$ is a closed subalgebra of $A$ containing that generated by $g, f$ and $f^{-1}$. Then if $U$ is any simply connected component of $g(M) \backslash g(\partial), \pi_{1}^{-1} U \cap \sigma_{B}(g, f)$ has the "analytic structure" given in Theorem 1.2.

To prove the lemma we note that Wermer's subharmonicity theorem asserts

$$
u_{+}: \zeta \longrightarrow \sup \log \left|f\left(g^{-1}(\zeta)\right)\right|
$$

is subharmonic on $g(M) \backslash g(X)$ (which of course includes any component of $C \backslash g(X)$ it meets [4, 8]), as is

$$
u_{-}: \zeta \longrightarrow-\inf \log \left|f\left(g^{-1}(\zeta)\right)\right| \text {, }
$$

since the result also applies to $f^{-1}$. Consequently $u_{+}+u_{-}$is a nonnegative subharmonic function on $g(M) \backslash g(X)$, which, by compactness of $M$ and our hypothesis, has $\varlimsup_{\zeta \rightarrow z}\left(u_{+}+u_{-}\right)(\zeta)=0$ for each $z \epsilon$ $\partial(g(M) \backslash g(X)) \subset g(X)$, and so is nonpositive by the maximum principle. Hence $u_{+}+u_{-} \equiv 0$ and thus $\left|f g^{-1}(\zeta)\right|$ is a singleton for each $\zeta \in g(M)$.

Now compactness of $M$ guarantees continuity of $u: \zeta \rightarrow \log \left|f\left(g^{-1}(\zeta)\right)\right|$, and since $u=u_{+}=-u_{-}, u$ is both sub- and superharmonic, so the lemma is proved.

In the setting of Theorem 1.2, $u$ has a well defined (and up to a constant, unique) continuous harmonic conjugate $v$ on $U$, so $u+i v$

1) Any element of $\hat{g}^{-1}(\zeta)$ is represented on $B$ by a nonnegative measure $\lambda$ carried by $X$, and $\lambda$ must also be carried by $\hat{g}^{-1}(\zeta)$ as one sees by applying it to $h^{n}$ and letting $n \rightarrow \infty$, where $h$ is our peaking function. Thus sup $\left|\hat{b}\left(\hat{g}^{-1}(\zeta)\right)\right| \leqq \sup \mid \hat{b}\left(\hat{g}^{-1}(\zeta) \cap X\right)=$ $\sup \left|b\left(g^{-1}(\zeta) \cap X\right)\right|$. 
is analytic on $U$. For any closed disc $D \subset U$ we thus have $(u+i v) \circ$ $g \in\left(A \mid g^{-1}(D)\right)^{-}$, so $h=f e^{-(u+i v) \circ g}$ is an invertible in that uniform algebra, while by local maximum modulus $g^{-1}(\partial D)$ forms a boundary for the algebra. Evidently $|h| \equiv 1$ on $g^{-1}(U)$ since $u(\zeta) \equiv \log \left|f\left(g^{-1}(\zeta)\right)\right|$, and if we take $\zeta \in D^{0}$ and let $m \in g^{-1}(\zeta)$, with $\lambda$ a measure on $g^{-1}(\partial D)$ representing $m$ then

$$
h(m)=\int h d \lambda
$$

and $|h| \equiv 1$ imply $h \equiv h(m)$ on the (closed) support of $\lambda$, which is mapped by $g$ onto the support of ${ }^{2)} g^{*} \lambda$. But $g^{*} \lambda$ is a measure on $\partial D$ which represents $\zeta$ on the disc algebra $A(D)$, so it necessarily has support all of $\partial D$. Thus $\partial D \subset g\left(h^{-1}(h(m))\right)$, and, since we can apply the same argument to any closed subdisc having $\zeta$ in its interior, we conclude that $D \subset \boldsymbol{g}\left(h^{-1}(h(m))\right)$. Hence

$$
g\left(h^{-1}(z)\right)=D \text { for all } z \text { in } K=h\left(g^{-1}(\zeta)\right) \subset T^{1} .
$$

In fact the compact set $K$ is independent of $\zeta \in D^{0}$, since if $\zeta^{\prime} \in D^{0}$ and $z \in K$ we have an $m^{\prime} \in h^{-1}(z)$ for which $g\left(m^{\prime}\right)=\zeta^{\prime}$ by (3), so $m^{\prime} \in g^{-1}\left(\zeta^{\prime}\right)$ and thus $z \in h\left(g^{-1}\left(\zeta^{\prime}\right)\right)$. Consequently from the connectedness of $U$ we have $K$ independent of our choice of the dise $D$ in $U$ as well.

So far we have not used the fact that $f$ and $g$ separate $M$. However for $V$ our relatively compact subdomain of $U$ and $B$ the closed subalgebra $\left(A \mid g^{-1}\left(V^{-}\right)\right)^{-}$of $C\left(V^{-}\right)$, since $f=h e^{(u+i v) \circ g}$ that hypothesis implies $g$ and $h$ separate $g^{-1}\left(V^{-}\right)=M_{B}$. Thus for each $z \in K, g \mid\left(h^{-1}(z) \cap g^{-1}\left(V^{-}\right)\right)$is $1-1$, and so maps its domain homeomorphically onto its range, which because of (3) must be $V^{-}$, the smallest compact set containing all closed dises $D \subset V$.

Evidently then $g^{-1}\left(V^{-}\right)$is homeomorphic to $V^{-} \times K$ via the map $(g, h)$. Moreover each element of our algebra is analytic on $V \times\{z\}, z \in K$ (or on $\left.h^{-1}(z)\right)$; more precisely $a \circ\left(g \mid h^{-1}(z)\right)^{-1}$ is analytic on $V$ for $a \in A$ : for we know $g^{-1}(\partial V)$ provides a boundary for $B$ by local maximum modulus, so since $h^{-1}(z) \cap g^{-1}\left(V^{-}\right)$is a peak set for $B$ (since $h \in B$ has unit modulus), $B \mid\left(h^{-1}(z) \cap g^{-1}\left(V^{-}\right)\right.$) is a closed algebra for which $h^{-1}(z) \cap g^{-1}(\partial V)$ provides a boundary exactly as in our observation before 1.3 (cf. footnote 1). In particular for $V^{-}=D$, a closed disc in $U$, we have $h^{-1}(z) \cap g^{-1}(D)$ a disc for which $g$ will provide a coordinate while $B$ restricted to this disc must have $h^{-1}(z) \cap g^{-1}(\partial D)$ as a boundary; thus the elements of $B$ (and so of $A$ ) are analytic functions of $g$ thereon by Wermer's

2) $g^{*} \lambda$ denotes the natural image measure: $g^{*} \lambda(E)=\lambda\left(g^{-1}(E)\right)$. 
maximality theorem [4]. Our proof is now complete.

To obtain Corollary 1.3, note that by the remarks immediately preceding it we can apply our lemma to any algebra $B$ as described and $M=M_{B}$; the preceding proof applies to show everything but the (lacking) 1 - 1-ness of our map of $M_{B} \cap \hat{g}^{-1}\left(V^{-}\right)=\hat{g}^{-1}\left(V^{-}\right)$onto $V^{-} \times K$, and of course $\sigma_{B}(g, f)$ is precisely the right quotient space to yield a homeomorphism.

When $U$ is not simply connected we can apply our argument locally, and evidently analytic continuation will allow us to regard $g^{-1}(U) \subset M_{A}$ as a product of a subset of $T^{1}$ and the Riemann surface provided by our continuation of $u+i v$ through $U$; that the correspondence is nontopological can be noted from the following example.

1.4. Let $X=\left\{(z, w) \in C^{2}:|z|=|w|=1\right.$, or $\left.|z|=r,|w|=r^{\sqrt{2}}\right\}$, $(1>r>0)$ and let $A$ be the closed algebra of $C(X)$ generated by $z, z^{-1}, w$ and $w^{-1}$. Then the spectrum of $A$ is $\left\{(z, w):|z|^{\sqrt{2}}=|w|\right.$, $r \leqq|z| \leqq 1\}$, as is well known; with $g=z$ and $f=w$, we obtain the familiar analytic structure in which $M \backslash X$ is the union of the graphs $z \rightarrow\left(z, e^{i \theta} z^{\sqrt{2}}\right)$ over $r<|z|<1$. (For $\left|f\left(g^{-1}(z)\right)\right| \equiv 1\left(\right.$ resp. $\left.\equiv r^{\sqrt{2}}\right)$ for $|z|=1$ (resp. $|z|=r$ ), so $u(z)=\sqrt{2} \log |z|$, whence $u+i v(z)=$ $\sqrt{2} \log z$ and $h=z^{-\sqrt{2}} f=w z^{-\sqrt{2}}$, so $h^{-1}\left(e^{i \theta}\right)$ is defined by $\left.w=e^{i \theta} z^{\sqrt{2}}\right)$.

1.5. In some situations even when $U$ is not simply connected we can arrive at a single valued harmonic conjugate for $u$, and thus the simple structure over $U$ afforded by Theorem 1.2. These follow from the simple observation that as we analytically continue $u+i v$ in $U, K$ never varies: "if $D_{1}$ is any disc centered at $\zeta_{1} \in D$ and $v$ is defined on $D_{1}$ so as to give (of course) the original value at $\zeta_{1}$, then for $m \in g^{-1}\left(\zeta_{1}\right), h_{1}(m)=f(m) \exp \left(-(u+i v)\left(\zeta_{1}\right)\right)=h(m)$, whence $h_{1}\left(g^{-1}\left(\zeta_{1}\right)\right)=h\left(g^{-1}(\zeta)\right)=K$. For one thing, this implies that if $p$ is the period of $v$ around some closed curve $\gamma$ in $U$ then

$$
e^{i p} K=K
$$

for another, we always have a well defined $h$ on $g^{-1}(U)$ with $h\left(g^{-1}(\zeta)\right)=K, \zeta \in U$, and, for $e^{i \theta} \in K, g\left(h^{-1}\left(e^{i \theta}\right)\right)=U$.

Consequently we can sometimes conclude $p=0$, as, for example in

Corollary 1.6. Suppose $g, f$ and $A$ are as in Theorem 1.2, and $U$ is a component of $g(M) \backslash g(X)$ whose boundary includes a point $\zeta_{0}$ for which $f\left(g^{-1}\left(\zeta_{0}\right)\right)$ has no nonvoid subset invariant under 
a nontrivial rotation. Then $g^{-1}(U)$ has the form given in Theorem 1.2 .

On $h^{-1}\left(e^{i \theta}\right)$ (for $\left.e^{i \theta} \in K\right)$ we have $f=e^{i \theta} e^{(u+i v) \circ g}$ and since $g\left(h^{-1}\left(e^{i \theta}\right)\right)=$ $U$, if $w$ is some cluster value of the (multiple valued) function $e^{u+i v}$ at $\zeta_{0}$ then $w$ appears as a cluster value of $e^{(u+i v) \cdot g} \mid h^{-1}\left(e^{i \theta}\right)$ at some $m_{0}$ in $h^{-1}\left(e^{i \theta}\right)^{-} \cap g^{-1}\left(\zeta_{0}\right)$. Thus $w e^{i \theta} \in f\left(g^{-1}\left(\zeta_{0}\right)\right)$ for each $e^{i \theta}$ in $K$, so $w \cdot K \subset f\left(g^{-1}\left(\zeta_{0}\right)\right)$, whence $e^{i p} w K=w K \subset f\left(g^{-1}\left(\zeta_{0}\right)\right)$ and we conclude $e^{i p}=1$ for each period because of our hypothesis on $f\left(g^{-1}\left(\zeta_{0}\right)\right)$.

Instead of starting with an algebra $A$ we can begin with a compact subset $X$ of $C^{2}$ (which is where $M_{A}$ lies in 1.2) and more or less rephrase 1.6 as follows, where $\mathscr{R}$ is some collection of rational functions on $\boldsymbol{C}$ including the identity function $z$ itself.

Corollary 1.7. Suppose $\left|\pi_{1}^{-1}(z)\right|$ is a singleton for each $z \in \pi_{1} X$, where $\pi_{1}: X \rightarrow C$ is projection onto the first coordinate, and suppose $\pi_{1} X$ is the minimal boundary of the subalgebra of $C\left(\pi_{1} X\right)$ generated by $\mathscr{R}$. Suppose $0 \notin \pi_{2} X$ and let $M$ be the spectrum of the algebra $A$ in $C(X)$ generated by $\mathscr{R}, w, 1 / w$. If $U$ is a component of $\hat{\pi}_{1} M \backslash$ $\pi_{1} X$ whose boundary includes a $z_{0}$ with $\pi_{2}\left(\pi_{1}^{-1}\left(z_{0}\right)\right)$ having no nonvoid subset invariant under a nontrivial rotation, then $\hat{\pi}_{1}^{-1}(U)$ has the form given in 1.2. (Here $\hat{\pi}_{1}$ is the first coordinate projection on $M$, viewed as a subset of $C^{2}$.)

We only have to note that as in the remark before $1.3, r_{z}=$ $\left|\pi_{1}^{-1}(z)\right|$ a singleton for $z$ in $\pi_{1} X$ implies $\left|\hat{\pi}_{1}^{-1}(z)\right|$ is also (for the same $z$ ), while $\hat{\pi}_{1}^{-1}\left(z_{0}\right)=\pi_{1}^{-1}\left(z_{0}\right)$ since $\pi_{1}^{-1}\left(z_{0}\right)$ provides a boundary for the closed algebra $A \mid \hat{\pi}_{1}^{-1}\left(z_{0}\right)$ (as in our remark) which is necessarily $C\left(\pi_{1}^{-1}\left(z_{0}\right)\right)$ since $\pi_{1}^{-1}\left(z_{0}\right)$ is a proper subset of the circle of radius $r_{z_{0}}$. Now Corollary 1.6 applies.

Actually 1.7 does provide some improvement since almost all hypotheses refer to $X$ and not $M$.

Rather than assume $z$ is invertible, we can assume $\pi_{1}^{-1}(z) \cap X$ is never a full circle. A special case is

CoROLlaRY 1.8. Suppose the compact set $X \subset C^{2}$ has the following properties:

(i) $\pi_{1} X$ lies in the boundary of the unbounded component ${ }^{3)}$ of $C \backslash \pi_{1} X$,

(ii) $\pi_{2} X$ does not separate 0 and $\infty$,

3) This does not imply $\pi_{1} X$ has connected interior; cf. Kerekjarto, Topologie I, Springer, 1923, frontespiece. 
(iii) $\left|\pi_{1}^{-1}(z) \cap X\right|$ is a singleton for each $z$ in $\pi_{1} X$, with $\pi_{1}^{-1}(z) \cap$ $X$ not a full circle,

where $\pi_{i}$ now is the projection of $C^{2}$ onto the ith coordinate. If $\hat{X}$ is the polynomial hull of $X$ and, for some component $U$ of $C \backslash \pi_{1} X$ we have a $z_{0} \in \partial U$ with $\pi_{1}^{-1} z_{0} \cap X$ a singleton, then $\pi_{1}^{-1} U \cap \hat{X}$ is the graph of a function holomorphic on $U$, or void.

Suppose the set nonvoid. We take $P(X)$ as our algebra, $g=z$ and $f=w$, and (i) implies each $z$ in $\pi_{1} X$ is a peak point for $P\left(\pi_{1} X\right)$ (since that is a dirichlet algebra [5]), so again $A=P(X) \mid \pi_{1}^{-1}(z) \cap \hat{X}$ is closed with boundary contained in $\pi_{1}^{-1}(z) \cap X$. Since $\pi_{1}^{-1}(z) \cap X$ is not a full circle $A=C\left(\pi_{1}^{-1}(z) \cap X\right)$ and $M_{A}=\pi_{1}^{-1}(z) \cap X$, so $\pi_{1}^{-1}(z) \cap$ $\hat{X}=\pi_{1}^{-1}(z) \cap X$ as before, and (iii) holds with $X$ replaced by $\hat{X}$, so $\left|f\left(g^{-1}(\zeta)\right)\right|$ is a singleton for $\zeta$ in $g(\hat{X})$ by 1.1. Since (ii) implies $f=w$ is invertible in $P(X)$ while $\pi_{1}^{-1} z_{0} \cap X$ a singleton implies our set $K$ must be a singleton $\left\{e^{i \theta}\right\}$ (since for any cluster point $w$ of $e^{u+i v}$ at $z_{0}$ we have $w K \subset \pi_{2}\left(\pi_{1}^{-1}\left(z_{0}\right)\right)$, a singleton, as in the proof of $1.6)$, and so $u+i v$ single valued, we obtain $f=e^{i \theta} e^{(u+i v) g}$, or $w=$ $e^{i \theta} e^{(u+i v)(z)}$ over $U$.

1.9. Some of the preceding holds even when $f$ is not invertible, provided $\left|f\left(g^{-1}(\zeta)\right)\right|$ rather strongly approaches constancy as $\zeta$ approaches a value for which $0 \in f\left(g^{-1}(\zeta)\right)$ : for $E=\left\{\zeta \in C: 0 \in f\left(g^{-1}(\zeta)\right)\right\}$ we assume that

$$
\lim _{\substack{\zeta \in U \backslash E \\ \zeta \rightarrow \zeta_{0}}} \frac{\sup \left|f\left(g^{-1}(\zeta)\right)\right|}{\inf \left|f\left(g^{-1}(\zeta)\right)\right|}=1, \zeta_{0} \in \partial E
$$

In particular then $0 \in f\left(g^{-1}(\zeta)\right)$ implies $\{0\}=f\left(g^{-1}(\zeta)\right)$ if $E$ is nowhere dense, and, in the setting of Lemma 1.1, $u_{+}$and $u_{-}$are again subharmonic on $U \backslash E, u_{+}+u_{-} \geqq 0$, and (5) implies $\overline{\lim }_{\zeta \rightarrow \zeta_{0} \zeta \in U \backslash E}\left(u_{+}+u_{-}\right)(\zeta) \leqq$ 0 for $\zeta_{0}$ in $\partial E$. So this inequality applies at each $\zeta_{0}$ in $\partial(U \backslash E) \subset$ $\partial U \cup \partial E$, and $u_{+}+u_{-} \equiv 0$ on $U \backslash E$. Now $u=u_{+}=-u_{-}$is harmonic on $U \backslash E$ again, but even with $U$ simply connected we can obtain a multiple valued extension of $u+i v$ to $U \backslash E$; of course at least locally we again can regard $g^{-1}(D)$ as a product and $f$ as an analytic function of $g$ on slices. However in at least one instance matters are quite simple.

Corollary 1.10. Suppose $f, g \in A$ satisfy (1) and (5), where $U$ is a component of $g(M) \backslash g(X)$, and separate $M$.

If, for some $\zeta_{0} \in U \backslash E$, card $f\left(g^{-1}\left(\zeta_{0}\right)\right)=n<\infty$ then there is a bounded holomorphic function $H$ on $U$ and a factorization $n=k \cdot l$ so that $g^{-1}(U)$ is the union of $l$ copies of the Riemann surface of 
$H^{1 / k}$ over $U$ (all identified over the branch points $H^{-1}(0)$ ) on which the elements of $A$ are holomorphic.

Here, since card $f\left(g^{-1}\left(\zeta_{0}\right)\right)=\operatorname{card} g^{-1}\left(\zeta_{0}\right)=\operatorname{card} h\left(g^{-1}\left(\zeta_{0}\right)\right)=\operatorname{card} K$, and $K=e^{i p} K$ by (4) for each period $p$ of $v$, the $e^{i p}$ must form the group $G$ of $k$ th roots of unity for some $k$, and $K$ the union of $l$ of its cosets. Consequently $H=e^{k(u+i v)}$ is a single valued holomorphic function on $U \backslash E=U \backslash H^{-1}(0)$ which is continuous on $U$, so holomorphic on $U$ by Rado's theorem [3,5]. If $K=\left\{e^{i \theta_{1}}, \cdots, e^{i \theta_{l}}\right\} G$ then on $h^{-1}\left(e^{i \theta_{j}}\right)$ we have

$$
f^{k}=e^{i k \theta_{j}} H \circ g
$$

and, via the map $(g, f)$ we can identify that part of $g^{-1}(U \backslash E)$ with a part of the Riemann surface defined by $w^{k}=e^{i k \theta_{j}} H(z)$; indeed since $K$ is independent of our $\zeta$ in $U \backslash E$, card $g^{-1}(\zeta)$ is constant over $U \backslash E$, so that by cardinality it must yield all of the Riemann surface for each $j$ (and in fact $e^{i k \theta_{j}} \neq e^{i k \theta_{j}}$ for $j_{1} \neq j_{2}$ ). Evidently then since $f(m) \rightarrow 0$ as $g(m)$ in $U \backslash E$ tends to $\zeta_{0} \in E=H^{-1}(0)$ we can conclude $(g, f)$ maps $g^{-1}(U)$ continuously $1-1$ onto the union of our Riemann surfaces with identification of the branch points over $\zeta$ in $H^{-1}(0)$, and so is a homeomorphism over $g^{-1}\left(V^{-}\right)$for $V \subset U$. The final conclusion is clear.

We can reach an analogous conclusion if we simply know $f\left(g^{-1}\left(\zeta_{0}\right)\right)$ is not a full circle for some $\zeta_{0}$ in $U \backslash E$; then the group of $e^{i p}$ can only be the kth roots of unity for some $k$ since $e^{i p} K=K$, so we again have $f^{k}=h^{k} H \circ g$ for a holomorphic $H$, and $g^{-1}(U)$ appears as a product of $\left\{z^{k}: z \in K\right\}$ and the Riemann surface for $H^{1 / k}$ (with an identification over $H^{-1}(0)=E$ ). (Here one argues that along with the homeomorph of $D \subset U$ we originally obtain we have those corresponding to multiplication by the $e^{i p}$ instead of using cardinality.)

Finally there is one very special instance in which neither (1) or (5) is explicitly needed: when $f$ and $g$ are inner functions with bounded ratios. Let $D$ be the unit disc.

THEOREM 1.11. Suppose $f$ and $g$ separate $M$ and are noninvertible and of unit modulus on $X$. If $f / g$ and $g / f$ are bounded on $M \backslash f^{-1}(0)=M \backslash g^{-1}(0)$ then $g^{-1}\left(D^{0}\right)$ is a union of analytic discs on each of which $f$ is a multiple of $g$; more generally if, for some $\alpha>0,|f| /|g|^{\alpha}$ and $|g|^{\alpha} /|f|$ are bounded on $M \backslash f^{-1}(0)=M \backslash g^{-1}(0)$ then $g^{-1}\left(D^{0}\right)$ is the union of copies of the Riemann surface for $w=z^{\alpha}$ (with $f, g$ corresponding to $w, z$ ), all identified over the branch point. 
Because of the abstract Schwarz lemma [6, 4.8] one knows in the first case that $|f / g|$ and $|g / f|$ are bounded by their suprema over $X$, i.e., by (1). Thus $|f| \equiv|g|$ and we know $\left|f\left(g^{-1}(\zeta)\right)\right|$ is always a singleton, and

$$
u: \zeta \longrightarrow \log \left|f\left(g^{-}(\zeta)\right)\right|
$$

is subharmonic on $D^{\circ} \subset g(M)$ by 1.1 (or directly from Wermer's theorem of course). On the other hand $u$ is harmonic on the punctured disc $D^{0} \backslash\{0\}=D^{*}$ as we can see by applying 1.1 to $\left(A \mid g^{-1}\left(D_{0}\right)\right)^{-}$where $D_{0}$ is a closed disc in $D^{*}$, since $f$ restricts to an invertible in that algebra because $f^{-1}(0)=g^{-1}(0)$. Thus since $|u| \equiv 1$ on $\partial D$, for some $\beta \geqq 0$ we have $u(\zeta)=\beta \log |\zeta|, \zeta \in D^{0}$, whence $u+$ $i v(\zeta)=\beta \log \zeta$ and for $h=f e^{-(u+i v)_{0} g}=f g^{-\beta}$ we have by our standard argument each section $h^{-1}\left(e^{i \theta}\right)$ (for $e^{i \theta} \in K$ ) a copy of the Riemann surface for $w=z^{\beta}$; this is in fact a disc since boundedness of $|f / g|$ and $|f| /\left|g^{\beta}\right|$ force $\beta=1$.

In the second case we again have $|f| /|g|^{\alpha}$ and $|g|^{\alpha} /|f|$ bounded by their suprema over $X$ by virtue of a recent extension of the abstract Schwarz lemma [2, Lemma 3], and again one obtains $u(\zeta)=$ $\beta \log |\zeta|$; but now $\beta=\alpha$ follows from the boundedness of $|f| /|g|^{\beta}$ and $|f| /|g|^{\alpha}$, and Riemann surfaces really appear. (Instead of $f, g$ inner we could assume $0 \neq|f|=\mid g_{\mid}^{\mid \alpha}$ on $X$ and obtain the same conclusion over the component $U$ of $g(M) \backslash g(X)$ containing 0: for then $u(\zeta)-\alpha \log |\zeta|$ is bounded and harmonic on $U \backslash\{0\}$, hence has an harmonic extension to $U$ which vanishes on $\partial U$, whence $u(\zeta) \equiv$ $\alpha \log |\zeta|$ and our conclusion follows as before.)

2. Some remarks, Replacing $A$ by $\left(A \mid g^{-1}(U)^{-}\right)^{-}$we can trivially deal with the case in which (1) holds only on $g(X) \cap \partial U$. Similarly, although we have phrased our observations in terms of analytic structure we can equally well ignore structure and produce functional dependence when $f$ and $g$ do not separate $M$, or when $A$ is only presented as a subalgebra of $C(X)$. (For example, (as in 1.8) if $g \in A \subset C(X), f \in A^{-1}$ and $g(X)$ lies in the minimal boundary of $P(g(X))$ (as in 1.8(i)) while (1) holds with $f\left(g^{-1}(\zeta)\right)$ never a full circle and $f\left(g^{-1}(\zeta)\right)$ is a singleton for one $\zeta \in \partial U$ for $U$ a component of $C \backslash g(X)$ then either $U$ misses $\sigma_{A}(g)=\hat{g}(M)$ or $\hat{f}=H \circ \hat{g}$ on $\hat{g}^{-1}(U)$ for $H \in H^{\infty}(U)$.)

Because of an old observation of Brian Cole (cf. [3, 4.1]) there is an extended form of Wermer's subharmonicity theorem which will allow the replacement of the logarithmic and exponential functions in our considerations. The extended theorem might be called 
2.1. The Cole Version of Wermer's Theorem. Suppose $f, g \in$ $A, U$ is a component of $g(M) \backslash g(X)$ and $\phi$ is a real function subharmonic on a neighborhood of the polynomial hull $\widehat{f\left(g^{-1}(U)\right)}$. Then

$$
\psi: \zeta \longrightarrow \sup \phi\left(f\left(g^{-1}(\zeta)\right)\right)
$$

is subharmonic on $U$.

First, it is routine to see $\psi$ is upper semi-continuous: if $\zeta_{n} \rightarrow \zeta$ in $U, \psi\left(\zeta_{n}\right) \geqq c$ implies there is $m_{n} \in g^{-1}\left(\zeta_{n}\right)$ with $\phi\left(f\left(m_{n}\right)\right) \geqq c-1 / n$, so if $m$ is a cluster point of $\left\{m_{n}\right\}$, then $f(m)$ is one of $\left\{f\left(m_{n}\right)\right\}$; if $f\left(m_{n_{i}}\right) \rightarrow f(m)$ then since $\phi$ is u.s.c., $\phi(f(m)) \geqq \lim \phi\left(f\left(m_{n_{i}}\right)\right) \geqq c$.

Now let $\zeta_{0}$ be the center of a closed disc $D \subset U$, let $m_{0} \in g^{-1}\left(\zeta_{0}\right)$ have $\phi\left(f\left(m_{0}\right)\right)=\psi\left(\zeta_{0}\right)$, and let $\lambda$ be a Jensen measure for $m_{0}$ carried by $g^{-1}(\partial D)$. Because of the Riesz decomposition theorem we know there is a nonnegative measure $\mu$ carried by a neighborhood of $\widehat{f\left(g^{-1}(U)\right)}$ with

$$
\phi(w)=\int \log |w-z| \mu(d z)
$$

for $w$ near $f\left(g^{-1}(U)\right)$, so

$$
\begin{aligned}
\psi\left(\zeta_{0}\right) & =\sup \phi\left(f\left(g^{-1}\left(\zeta_{0}\right)\right)\right)=\phi\left(f\left(m_{0}\right)\right)=\int \log \left|f\left(m_{0}\right)-z\right| \mu(d z) \\
& \leqq \iint \log |f(m)-z| \lambda(d m) \mu(d z)=\int \phi(f(m)) \lambda(d m) \\
& \leqq \int \sup \phi\left(f\left(g^{-1}(g(m))\right)\right) \lambda(d m)=\int \psi(g(m)) \lambda(d m) \\
& =\int \psi(\zeta) g^{*} \lambda(d \zeta) .
\end{aligned}
$$

But $g^{*} \lambda$ is a measure on $\partial D$ representing $\zeta_{0}$ on the disc algebra, and so coincides with the normalized boundary measure $d \theta / 2 \pi$, whence $\psi$ is subharmonic, completing our proof.

Of course the proof is simply the combination of Cole's observation on the applicability of Riesz's theorem to the theory of Jensen measures and the well known folk proof of Wermer's theorem, but the result allows us to repeat our observations in a broader context. Indeed if we now suppose $\phi=R e F$ for $F$ holomorphic near the spectrum of $f \in A$ while $\phi\left(f\left(g^{-1}(\zeta)\right)\right)$ is a singleton for each $\zeta \in g(X)$ then the argument of lemma 1.1 shows the same is true for $\zeta$ in $g(M) \backslash g(X)$ and that

$$
u: \zeta \longrightarrow \phi\left(f\left(g^{-1}(\zeta)\right)\right)
$$


is harmonic on that set and continuous on $g(M)$. Let $v$ be the harmonic conjugate. Again one concludes for $H=u+i v$ that on a simply connected component $U$ of $g(M) \backslash g(X)$ we have $h=$ $\exp (H \circ g-F \circ f)$ unimodular on $g^{-1}(U)$ since $\operatorname{Re} H \circ g=u \circ g=\phi \circ f=$ Re $F \circ f$, and also conclude that $K=h\left(g^{-1}(\zeta)\right)$ is independent of $\zeta$ while $g\left(h^{-1}(z)\right)=U$; but now our relation between $f$ and $g$ on the sections $h^{-1}(z)$ is implicit: $H \circ g=F \circ f+\log h^{-1}(z)$. (For $F=\log$ we have our original case, since clearly $F$ can be multivalued as long as $\operatorname{Re} F$ is single valued.) Of course if $F$ has a single valued inverse we can find $f$ explicitly and the situation exactly parallels the original.

Use of Jensen measures $\lambda$ and their images $g^{*} \lambda$ on $g(X)$ as in the preceding proof also show that in all our results we can weaken (1) to

$\left|f\left(g^{-1}(\zeta)\right)\right|$ is a singleton for all $\zeta \in g(X) \backslash E$, where $E$ is null for all Jensen measures for points in $g(M) \backslash g(X)$ on $R(g(M))$.

Indeed, given $U$, for any $\zeta_{0} \in g^{-1}(U)$ as in (6) we have

$$
\sup \log \left|f\left(g^{-1}\left(\zeta_{0}\right)\right)\right| \leqq \int \sup \log \left|f\left(g^{-1}(\zeta)\right)\right| g^{*} \lambda(d \zeta),
$$

where $\lambda$ is now a Jensen measure for $\left(A \mid g^{-1}(U)^{-}\right)^{-}$, carried by $g^{-1}(\partial U)$, for $m_{0} \in g^{-1}\left(\zeta_{0}\right)$ (which exists because of local maximum modulus); moreover $g^{*} \lambda$ is Jensen for $R(g(M))$, since $R(g(M)) \circ g \subset A$. Thus for $f \in A^{-1}$ we can conclude from ( $\left.1^{\prime}\right)$ that $u_{+}$and $u_{-}$, as defined in the proof of Lemma 1.1 , have $u_{+}+u_{-}=0$ on $\partial U \backslash E$, so (7) implies $\left(u_{+}+u_{-}\right)\left(\zeta_{0}\right) \leqq 0$ (hence $=0$ ), and $u$ is harmonic on $g(M) \backslash g(X)$. (Of course we do not obtain continuity at points of $E^{-}$.)

Because of [2, Lemma 1] (applied to the algebra $R(g(M))$, with $f$ the identity function) any Borel $E \subset g(X)$ of (inner logarithmic) capacity zero is appropriately Jensen null: thus our results hold with (1) replaced by

$$
\begin{aligned}
& \left|f\left(g^{-1}(\zeta)\right)\right| \text { is a singleton for each } \\
& \quad \zeta \in g(X) \backslash E \text {, where } E \text { is a Borel subset of capacity zero. }
\end{aligned}
$$

Finally there is an extension of sorts of Wermer's maximality theorem which I noted because of the observation in Lemma 1.1, but which follows most simply (as Brian Cole kindly pointed out to me) from the fact [1, Lemma 1] that the diameter function $\zeta \rightarrow$ diam $f\left(g^{-1}(\zeta)\right)$ is subharmonic on $g(M) \backslash g(X)$; this is another corollary of Wermer's subharmonicity theorem. 
THEOREM 2.2. Suppose $K \subset C$ is compact, $A$ is a closed subalgebra of $C(\partial K)$ containing $R(K) \mid \partial K$, and let $\pi: M_{A} \rightarrow K$ be dual to $R(K) \rightarrow A$ so $K_{0}=\pi M_{A}$ is the union of $\partial K$ and certain components of the interior $K^{0}$. If $\partial K$ is the minimal boundary for $R(K)$ (or just the Jensen boundary [5, p. 28], whose elements have unique Jensen measures) then

$$
R\left(K_{0}\right)\left|\partial K \subset A \subset A\left(K_{0}\right)\right| \partial K .
$$

In particular if $R\left(K_{0}\right)=A\left(K_{0}\right)$ for every union $K_{0}$ of $\partial K$ and interior components of $K$ then algebras on $\partial K$ containing $R(K) \mid \partial K$ are determined by their spectra, each corresponding to some $K_{0}$. I have no idea whether $\partial K$ being the Jensen boundary for $R(K)$ is essential for the result, but it provides the crucial fact that, for $z$ in $\partial K, \pi^{-1}(z)$ is a singleton, since any Jensen measure for $m \in \pi^{-1}(z)$ represents $z$ on $R(K)$. Now for $g(z) \equiv z, g \in A$, and for $f \in A$,

$$
\zeta \longrightarrow \operatorname{diam} \hat{f}\left(\hat{g}^{-1}(\zeta)\right)=\operatorname{diam} \hat{f}\left(\pi^{-1}(\zeta)\right)
$$

is a nonnegative subharmonic function on $^{4)} K_{0} \mid \partial K$ which has zero boundary values, so is identically zero. But then $\pi^{-1}(\zeta)$ is always a singleton, so $M_{A}$ and $K_{0}$ can be identified, while $\hat{f}$ is analytic on any disc in $K_{0} \mid \partial K$ by Wermer's maximality theorem so $\hat{f}$ is analytic on $K_{0} \mid \partial K$, and $f \in A\left(K_{0}\right)$. Thus $A \subset A\left(K_{0}\right) \mid \partial K$. On the other hand for $z_{0} \in K^{0} \backslash K_{0}$ we have $z \rightarrow z-z_{0}$ invertible in $A$, so $A \supset R\left(K_{0}\right) \mid \partial K$ since $R\left(K_{0}\right) \mid \partial K$ is spanned by $R(K)$ and the inverses of such functions.

Corollary 2.3. If $R(K)=A(K)$ has $\partial K$ as minimal boundary and $K_{0}=\pi M_{A}$ is obtained by deletion of finitely many interior components of $K$ then $A=R\left(K_{0}\right) \mid \partial K$.

Because of 2.2 one only has to see $R(K)=A(K)$ is preserved when one deletes one component $U$ of $K^{0}$. But that follows from Vituskin's criteria for this equality: if $x \in \partial K$ does not lie in $\partial U$ then (v) of [4, VIII 8.2] holds with $K$ replaced by $K \backslash U$ ( $r \delta$ should appear in the denominator for $\delta$ ), while if $z \in \partial U$ then for $0<\delta<$

4) To argue as in 1.1 instead, one notes that for $c$ complex with $|c|>\|f\|$, since $c+f \in A^{-1}$

$$
u(\zeta)=\sup \log \left|c+\hat{f}\left(\hat{g}^{-1}(\zeta)\right)\right|-\inf \log \left|c+\hat{f}\left(\hat{g}^{-1}(\zeta)\right)\right|
$$

is a nonnegative subharmonic function on $K_{0} / \partial K$ with zero boundary values, so $\equiv 0$. Evidently this implies $\hat{f}\left(\hat{g}^{-1}(\zeta)\right)$ is always a singleton. We can also see from the argument that it suffices to assume only that the minimal boundary for $R(K)$ carries all Jensen measures on $\partial K$ for points in $\left(K_{0}\right)^{0}$ : for then our $u$ vanishes on enough of $\partial K$ to insure $u \leqq 0$ on $\left(K_{0}\right)^{0}$, as in our argument following (7). Alternately, we could assume $\partial K$ is the union of the Jensen boundary and a Borel set of capacity zero. 
$1 / 2 \operatorname{diam} U, \Delta(z, \delta)^{\circ} \backslash(K \backslash U)$ contains an arc of diameter $\geqq \delta / 2$ (where $\Delta(z, \delta)$ is the disc of radius $\delta$, about $z)$ so that, as in [4, VIII 8.4], $\alpha\left(\Delta(z, \delta)^{\circ} \backslash(K \backslash U) \geqq(1 / 8) \delta \geqq(1 / 8) \alpha\left(\Delta(z, \delta) \backslash(K \backslash U)^{0}\right)\right.$, which implies (v) again.

\section{REFERENCES}

1. B. Aupetit and J. Wermer, Capacity and uniform algebras, J. Functional Analysis, 28 (1978), 386-400.

2. B. J. Cole and I. Glicksberg, Jensen measures and a theorem of Radó, J. Funct. Anal., 35 (1980), 26-41.

3. T. W. Gamelin, The polynomial hulls of certain subsets of $\boldsymbol{C}^{2}$, Pac. J. Math., 61 (1975), 129-142.

4. — Uniform Algebras, Prentice Hall, Englewood Cliffs, N. J., 1969.

5. — Uniform algebras and Jensen Measures, London Math. Soc. Lecture Notes, 32, Cambridge U. Press, 1978.

6. I. Glicksberg, Maximal algebras and a theorem of Radó, Pac. J. Math., 14 (1964), 919-941.

7. J. Wermer, Subharmonicity and hulls, Pac. J. Math., 58 (1975), 283-290.

8. —_ Banach Algebras and Several Complex Variables, 2nd Ed., Springer, N. Y., 1976.

Received January 19, 1980. Work supported in part by the NSF.

UNIVERSITY OF WASHINGTON

Seatrle, WA 98195 


\section{PACIFIC JOURNAL OF MATHEMATICS}

\section{EDITORS}

DONALD BABBITT (Managing Editor)

University of California

Los Angeles, CA 90024

Hugo RossI

University of Utah

Salt Lake City, UT 84112

C. C. MOORE and ANDREW OGG

University of California

Berkeley, CA 94720
J. DugundjI

Department of Mathematics

University of Southern California

Los Angeles, CA 90007

R. FINN and J. MILGRAM

Stanford University

Stanford, CA 94305

\section{ASSOCIATE EDITORS}
R. ARENS
E. F. BECKENBACH
B. H. NeUmanN
F. WOLF
K. YosHIDA

\section{SUPPORTING INSTITUTIONS}

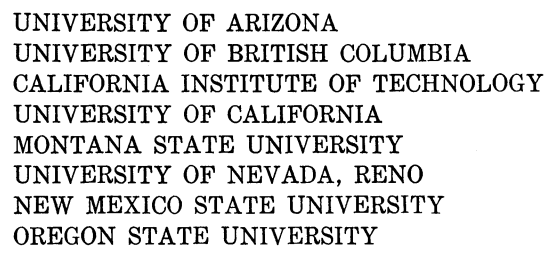

UNIVERSITY OF ARIZONA

UNIVERSITY OF BRITISH COLUMBIA CALIFORNIA INSTITUTE OF TECHNOLOGY

UNIVERSITY OF CALIFORNIA

MONTANA STATE UNIVERSITY

UNIVERSITY OF NEVADA, RENO

NEW MEXICO STATE UNIVERSITY OREGON STATE UNIVERSITY

\author{
UNIVERSITY OF OREGON \\ UNIVERSITY OF SOUTHERN CALIFORNIA \\ STANFORD UNIVERSITY \\ UNIVERSITY OF HAWAII \\ UNIVERSITY OF TOKYO \\ UNIVERSITY OF UTAH \\ WASHINGTON STATE UNIVERSITY \\ UNIVERSITY OF WASHINGTON
}

The Supporting Institutions listed above contribute to the cost of publication of this Journal, but they are not owners or publishers and have no responsibility for its content or policies.

Mathematical papers intended for publication in the Pacific Journal of Mathematics should be in typed form or offset-reproduced, (not dittoed), double spaced with large margins. Please do not use built up fractions in the text of the manuscript. However, you may use them in the displayed equations. Underline Greek letters in red, German in green, and script in blue. The first paragraph or two must be capable of being used separately as a synopsis of the entire paper. Please propose a heading for the odd numbered pages of less than 35 characters. Manuscripts, in triplicate, may be sent to any one of the editors. Please classify according to the scheme of Math. Reviews, Index to Vol. 39. Supply name and address of author to whom proofs should be sent. All other communications should be addressed to the managing editor, or Elaine Barth, University of California, Los Angeles, California, 90024.

50 reprints to each author are provided free for each article, only if page charges have been substantially paid. Additional copies may be obtained at cost in multiples of 50 .

The Pacific Journal of Mathematics is issued monthly as of January 1966. Regular subscription rate: $\$ 102.00$ a year (6 Vols., 12 issues). Special rate: $\$ 51.00$ a year to individual members of supporting institutions.

Subscriptions, orders for numbers issued in the last three calendar years, and changes of address shoud be sent to Pacific Journal of Mathematics, P.O. Box 969, Carmel Valley, CA 93924, U.S.A. Old back numbers obtainable from Kraus Per!odicals Co., Route 100, Millwood, NY 10546.

\section{PUBLISHED BY PACIFIC JOURNAL OF MATHEMATICS, A NON-PROFIT CORPORATION}

Printed at Kokusai Bunken Insatsusha (International Academic Printing Co., Ltd.). 8-8, 3-chome, Takadanobaba, Shinjuku-ku, Tokyo 160, Japan.

Copyright (C) 1981 by Pacific Jounal of Mathematics Manufactured and first issued in Japan 


\section{Pacific Journal of Mathematics}

Vol. 94, No. $2 \quad$ June, 1981

Thomas E. Armstrong and William David Sudderth, Nearly strategic

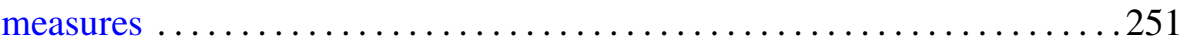

John J. Buoni, Artatrana Dash and Bhushan L. Wadhwa, Joint Browder

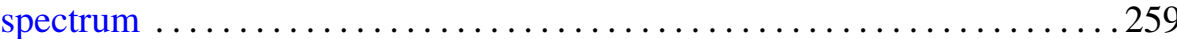

Jack Paul Diamond, Hypergeometric series with a $p$-adic variable . .......265

Raymond Frank Dickman, Jack Ray Porter and Leonard Rubin,

Completely regular absolutes and projective objects ............ 277

James Kenneth Finch, On the local spectrum and the adjoint ........... 297

Benno Fuchssteiner, An abstract disintegration theorem ............ 303

Leon Gerber, The volume cut off a simplex by a half-space $\ldots \ldots \ldots \ldots 311$

Irving Leonard Glicksberg, An application of Wermer's subharmonicity

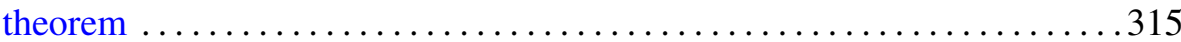

William Goldman, Two examples of affine manifolds ............... 327

Yukio Hirashita, On the Weierstrass points on open Riemann surfaces .....331

Darrell Conley Kent, A note on regular Cauchy spaces ............. 333

Abel Klein and Lawrence J. Landau, Periodic Gaussian

Osterwalder-Schrader positive processes and the two-sided Markov

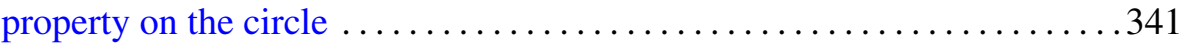

Brenda MacGibbon, $\mathscr{K}$-Borelian embeddings and images of Hausdorff

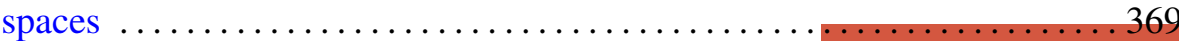

John R. Myers, Homology 3-spheres which admit no PL involutions . . . . . 379

Boon-Hua Ong, Invariant subspace lattices for a class of operators . . . . . 385

Chull Park, Representations of Gaussian processes by Wiener processes . . . 407

Lesley Millman Sibner and Robert Jules Sibner, A sub-elliptic estimate

for a class of invariantly defined elliptic systems $\ldots \ldots \ldots \ldots \ldots \ldots .417$

Justin R. Smith, Complements of codimension-two submanifolds. III.

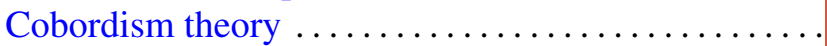

William Albert Roderick Weiss, Small Dowker spaces

David J. Winter, Cartan subalgebras of a Lie algebra and its ideals. II ... 